\title{
Two Problems of Time Entering Respectively the Relativistic Mechanics and Electron Transport in Quantum Theory
}

\author{
Stanisław Olszewski \\ Institute of Physical Chemistry, Polish Academy of Sciences, Warsaw, Poland \\ Email: solszewski@ichf.edu.pl
}

How to cite this paper: Olszewski, S. (2020) Two Problems of Time Entering Respectively the Relativistic Mechanics and Electron Transport in Quantum Theory. World Journal of Mechanics, 10, 186-197. https://doi.org/10.4236/wjm.2020.1010013

Received: August 21, 2020

Accepted: October 19, 2020

Published: October 22, 2020

Copyright $\odot 2020$ by author(s) and Scientific Research Publishing Inc. This work is licensed under the Creative Commons Attribution International License (CC BY 4.0).

http://creativecommons.org/licenses/by/4.0/

\begin{abstract}
In the relativistic mechanics, we calculate a minimal distance between the time scale of a one-dimensional motion having a larger velocity and the time scale of a similar motion with a lower velocity. Concerning the quantum theory, we demonstrate that mechanical parameters entering the electron motion in the Bohr hydrogen atom can provide us with a correct size of the time interval entering the Joule-Lenz law for the emission energy between two neighbouring quantum levels of the atom.
\end{abstract}

\section{Keywords}

Time in the Special Relativity Theory, Time in the Quantum Theory of the Bohr Atom, Joule-Lenz Law for the Emission of Energy in the Atom

\section{c) (i) Open Access}

\section{Time and the Classical Physics; Introduction}

Evidently the time problem-raised as a consequence of the observation of any change-is as old as the humanity is. In the everyday life, we have a dawn, a day and a night, and this sequence does repeat infinitely indicating also the differences entering particular dawn-day-night sets. In classical physics, time became a parameter which allows us to distinguish a later event from an earlier event. The parameter is consequently incorporated as an independent variable entering the classical mechanics and its laws; see e.g. [1]. In the first step, in the Newtonian mechanics, time is a variable fully independent of the other physical parameters.

But the problem of invariance of the physical laws considered with respect to the motion properties of a physical system led-in a special relativity-to the time notion which became, first, the function of the velocity components of the moving body, next a function of the maximal velocity size attributed to the light. 
In the general relativity, the time interval became dependent also on other parameters than the body velocity, for example the body mass; see e.g. [2].

A separate problem which became of importance was the irreversibility of time. In fact, only one kind of the time change, that of a spontaneous increase of the time variable, could be postulated as valid in nature. A correspondence of this property with a systematic increase of entropy predicted by the second law of thermodynamics became here well-known and famous.

On the first level of the paper, no quantum effects come into play. Our aim is to study in Section 2 - 4 the problem of an extremal (minimal) time difference attainable by the time variables belonging to two different frames considered in the mechanics of special relativity. This problem seems to be neglected in the earlier calculations, but can be solved now in a rather simple way.

In the next step-Section 6, the time interval necessary for the electron transition between two neighbouring energy levels of the hydrogen atom is calculated in a semiclassical way. The interval is found to fit very well the Joule-Lenz law for the quantum electron transition. Electrodynamical repercussions of the law are also presented.

\section{Change of the Time Variable in Special Relativity Examined as a Function of the Particle Velocity}

A very simple approach to the theory of special relativity is based on a single position variable $z$ and the time variable $t$ of a moving particle [3]. This kind of motion can be called one-dimensional. If the particle motion is considered next in a system frames which move with a speed of $v$ in the direction of $z$, the particle coordinates $(z, t)$ are modified-according the well-known formulae of the Lorentz transformation-into the variables $\left(z^{\prime}, t^{\prime}\right)$ which are respectively:

$$
z^{\prime}=\frac{z-v t}{\left(1-\frac{v^{2}}{c^{2}}\right)^{1 / 2}}
$$

and

$$
t^{\prime}=\frac{t-\frac{v z}{c^{2}}}{\left(1-\frac{v^{2}}{c^{2}}\right)^{1 / 2}} .
$$

Our aim is to examine in some detail the time difference $t^{\prime}-t$ as a function of the speed $v$, especially the extremal properties of the mentioned difference become of interest.

The formula (2) implies that for the velocity and position parameters which satisfy the relations

$$
v>0, \quad z>0,
$$

the time variables $t^{\prime}$ and $t$ are referred together by the formula which gives

$$
t^{\prime}<t \text {. }
$$


Our aim is to demonstrate that relation (4) is limited only to an interval of $t^{\prime}-t$. Evidently the property (4) holds irrespectively of the size of the (positive) factor

$$
\left(1-\frac{v^{2}}{c^{2}}\right)^{1 / 2}
$$

entering the denominator in (2). In order to make the influence of the ratio

$$
v / c
$$

on our considerations approximately negligible, we may assume

$$
v \ll c \text {. }
$$

This implies in practice a replacement of (5) by the term very close to unity.

\section{Variational Approach to the Change of the Time Variable Due to the Lorentz Transformation}

The formulae (1) and (2) can be easily transformed into an expression having a difference between the variables $t^{\prime}$ and $t$ being more suitable for a variational treatment than (1) and (2) alone. On this step we do not apply the simplification presented in (7). By taking the square values of the expressions entering (1) and (2) we obtain from (2):

$$
t^{\prime 2}\left(1-\frac{v^{2}}{c^{2}}\right)=t^{2}-\frac{2 z v t}{c^{2}}+\frac{z^{2} v^{2}}{c^{4}}
$$

and from (1):

$$
\frac{z^{\prime 2}}{c^{2}}\left(1-\frac{v^{2}}{c^{2}}\right)=\left(z^{2}-2 z v t+v^{2} t^{2}\right) \frac{1}{c^{2}} .
$$

By subtracting (9) from (8) we obtain

$$
\left(t^{\prime 2}-\frac{z^{\prime 2}}{c^{2}}\right)\left(1-\frac{v^{2}}{c^{2}}\right)=t^{2}-\frac{z^{2}}{c^{2}}+\frac{v^{2}}{c^{2}}\left(\frac{z^{2}}{c^{2}}-t^{2}\right)=\left(t^{2}-\frac{z^{2}}{c^{2}}\right)\left(1-\frac{v^{2}}{c^{2}}\right)
$$

so we arrive at the equation

$$
t^{\prime 2}-t^{2}=\frac{z^{\prime 2}}{c^{2}}-\frac{z^{2}}{c^{2}}
$$

This formula can be also obtained on the basis of the reference [3].

In the next step we apply the approximation given in (7) so

$$
z^{\prime 2}=\frac{(z-v t)^{2}}{1-\frac{v^{2}}{c^{2}}} \approx(z-v t)^{2}=z^{2}-2 z v t+v^{2} t^{2} .
$$

In effect of (12) we obtain

$$
\frac{z^{\prime 2}}{c^{2}}-\frac{z^{2}}{c^{2}} \approx\left(-2 z v t+v^{2} t^{2}\right) \frac{1}{c^{2}} .
$$

The extremal properties of (13) with respect to the velocity $V$ can be easily examined. From the requirement 


$$
\frac{\mathrm{d}}{\mathrm{d} v}\left(-2 z v t+v^{2} t^{2}\right)=-2 z t+2 v t^{2}=0,
$$

an extremal value of the expressions in (11), or (13), occurs at

$$
2 v t^{2}=2 t z
$$

or

$$
t=t_{\min }=\frac{z}{v} .
$$

The second derivative of (13) with respect to $v$ gives

$$
\frac{\mathrm{d}^{2}}{\mathrm{~d} v^{2}}\left(-2 z v t+v^{2} t^{2}\right)=\frac{\mathrm{d}}{\mathrm{d} v}\left(2 v t^{2}\right)=2 t^{2}>0,
$$

therefore the formulae (15) and (15a) indicate a position of a minimum of (11) or (13).

Evidently at $t=0$ the expression in (13) becomes zero and-because of (11) - the same property applies to the left-hand side of (13), so in this case

$$
t^{\prime}=t=0 .
$$

A minimum value of (11) or (13) becomes

$$
\left(-2 z v t_{\min }+v^{2} t_{\min }^{2}\right) \frac{1}{c^{2}}=\left(-2 \frac{z^{2} v}{v}+v^{2} \frac{z^{2}}{v^{2}}\right) \frac{1}{c^{2}}=-\frac{z^{2}}{c^{2}}
$$

when the value of (15a) is substituted for $t$ in (13). This gives also

$$
t^{\prime 2}=t^{2}\left(1-\frac{v^{2}}{c^{2}}\right)
$$

On the other hand, for example for

$$
t=2 t_{\text {min }}=2(z / v),
$$

we obtain

$$
\left(z^{\prime 2}-z^{2}\right) \frac{1}{c^{2}}=\left[-2 v \frac{2 z}{v} z+v^{2}\left(\frac{2 z}{v}\right)^{2}\right] \frac{1}{c^{2}}=0,
$$

and also-because of (11)

$$
t^{\prime 2}-t^{2}=0 .
$$

Another example can be the case of

$$
t=3 t_{\min }=3(z / v) .
$$

This gives

$$
\left(z^{\prime 2}-z^{2}\right) \frac{1}{c^{2}}=\left[-2 v \frac{3 z}{v} z+v^{2}\left(\frac{3 z}{v}\right)^{2}\right] \frac{1}{c^{2}}=\frac{3 z^{2}}{c^{2}}=t^{\prime 2}-t^{2}>0 .
$$

\section{Special Behaviour of the Time Extremum $\left(t_{\min }\right)$ Obtained in (15a)}

In principle we look for an extremum with respect to $v$ of the expression 


$$
z^{\prime 2}-z^{2}=\frac{(z-v t)^{2}}{1-\frac{v^{2}}{c^{2}}}-z^{2}
$$

in which the denominator of $z^{\prime 2}$ can be represented by a series of the even powers of $v / c$ :

$$
\frac{1}{1-\frac{v^{2}}{c^{2}}}=1+\frac{v^{2}}{c^{2}}+\frac{v^{4}}{c^{4}}+\cdots
$$

Evidently-in the case of (7) - the powers entering (23) can give only small numbers.

A characteristic point is that when the series (23) is taken into account, the extremum value of $t_{\min }$ obtained before in (15a) for the case of neglected powers entering (23) does not change. This becomes evident if we note that expression (22) in its part dependent on $v$ is equal to a sum of

$$
(z-v t)^{2}
$$

identical with the expression examined on the right of (12) and leading to (15a), and expression

$$
(z-v t)^{2}\left(\frac{v^{2}}{c^{2}}+\frac{v^{4}}{c^{4}}+\cdots\right)
$$

But expression (25) is a sum of powers

$$
(z-v t)^{2} v^{2 n} \frac{1}{c^{2 n}}
$$

any of which gives in course of the differentiation process with respect to $v$ the result equal to zero:

$$
\frac{\mathrm{d}}{\mathrm{d} v}(z-v t)^{2} v^{2 n}=2(z-v t)(-t) v^{2 n}+(z-v t)^{2} 2 n v^{2 n-1}=0
$$

if we substitute $z=v t$ from (15) into expression (26). This fact makes position of the extremum value of (22) equal to that given by (12), therefore $t_{\min }$ becomes equal to (15a).

The second derivative of (25a) calculated with respect to $v$ gives

$$
\begin{aligned}
& \frac{\mathrm{d}^{2}}{\mathrm{~d} v^{2}}(z-v t)^{2} v^{2 n}=2 \frac{\mathrm{d}}{\mathrm{d} v}(z-v t)(-t) v^{2 n}+2 n \frac{\mathrm{d}}{\mathrm{d} v}(z-v t)^{2} v^{2 n-1} \\
& =2 t^{2} v^{2 n}>0
\end{aligned}
$$

if $z=v t$, so we attain again at (15a) a minimum of the $v$ dependence of the expression given in (22).

The expression

$$
t^{\prime 2}-t^{2}
$$

calculated at minimum becomes identical to that obtained in the absence of the ratio $v / c$ in the denominator of the square of $z-v t$ : 


$$
t^{\prime 2}-t^{2}=\left.\frac{1}{c^{2}} \frac{(z-v t)^{2}}{1-\frac{v^{2}}{c^{2}}}\right|_{t=\frac{z}{v}}-\frac{z^{2}}{c^{2}}=-\frac{z^{2}}{c^{2}}
$$

see (18). The differences from results obtained in Section 3 occur for $t=2 t_{\min }$ which gives

$$
t^{\prime 2}-t^{2}=\frac{z^{2}}{c^{2}}\left(\frac{1}{1-\frac{v^{2}}{c^{2}}}-1\right)
$$

but not zero obtained in (20) and (20a), for $t=3 t_{\min }$ we obtain

$$
t^{\prime 2}-t^{2}=\frac{z^{2}}{c^{2}}\left(\frac{4}{1-\frac{v^{2}}{c^{2}}}-1\right)
$$

but not the result from (21) and (21a), etc.

\section{Time and the Quantum Theory}

A discovery of the quantum effects in physics led to a new look on time; see e.g. [4]. In brief time ceased to be a welcomed variable in describing the physical phenomena. The first reason of that became a conviction that the time interval connected experimentally with an individual quantum process was much too short to be made compared adequately with the results of calculations done with the aid of the theory. Another reason was an evident abundance of the effects being of the same-or much similar-kind. Then an observation of a separate single effect was considered to be associated with an unrealistically high difficulty. In consequence the statistics similar to that given by the Boltzmann law has been applied [2] [5]. A supplementary reason of the lack of an individual time examination of the quantum effects was of a formal nature: the modern quantum theory was unable to construct an operator representing adequately the time; see e.g. [6] [7].

This situation made any theoretical description of the time-dependent change performed by a quantum system to be a rather uncertain task. In practice this meant that the quantum mechanics was reduced to a quantum statics: only stationary states of the atomic systems could be represented by the quantum theory. Simultaneously, the computational effort connected with an accurate description of such states was drastically complicated by an increase of the particle number involved in the calculations.

\section{Special Time Problem Entering the Quantum Theory of the Atom}

Historically the perfect success of the semiclassical Bohr theory obtained for the 
energy spectrum in the hydrogen atom dominated the development of the quantum theory [8] [9] [10]. In this section we try to apply that theory in calculating the time intervals necessary for the electron transitions in the hydrogen atom; the atom is evidently considered on a semiclassical level. The virial theorem for the electron energy in the hydrogen atom states that [11]

$$
2 E_{\text {kin }}+E_{\text {pot }}=0
$$

where $E_{\text {kin }}$ is the kinetic electron energy

$$
E_{\text {kin }}=\frac{1}{2} m v_{n}^{2}
$$

and

$$
E_{\mathrm{pot}}=-\frac{e^{2}}{r_{n}}
$$

is the potential electron energy on the same quantum level $n$. The electron speed on the level $n$ is [11]

$$
v_{n}=\frac{e^{2}}{n \hbar},
$$

whereas the radius of the circular orbit track corresponding to the level $n$ is [11]

$$
r_{n}=\frac{n^{2} \hbar^{2}}{m e^{2}}
$$

The above parameters provide us with the following result for the total electron energy on the level $n$ :

$$
E_{\mathrm{tot}}=E_{\mathrm{kin}}+E_{\mathrm{pot}}=-\frac{1}{2} m v_{n}^{2}=-\frac{1}{2} \frac{m e^{4}}{n^{2} \hbar^{2}} .
$$

Our aim is to calculate the time of the electron transfer from the level $n+1$ (less negative energy) to the level $n$ (more negative energy). This task can be approached in several ways. In the first step, we can apply the Joule-Lenz theorem developed earlier on the basis of a quantum approach to the classical Joule-Lenz dissipation energy (see [12] [13])

$$
\Delta E \Delta t=h,
$$

where

$$
\Delta E=E_{n+1}-E_{n}
$$

is the energy difference between the total electron energy on the levels $n+1$ and $n$ which are the nearest neighbouring quantum states in the atom. This kind of calculations was widely applied in examining the intensity of the emission spectra of the atomic hydrogen; see [13]-[17]. In the present case we refer to the result of the Joule-Lenz law in (37) again at the end of the present section.

A more direct approach to the emission time interval $\Delta t$ can be obtained, first, by considering the change of the total electron energy with the change of the electron velocity between the states $n+1$ and $n$. We obtain 


$$
\frac{\Delta E}{\Delta t}=-m v_{n} \frac{\Delta v_{n}}{\Delta t}=\frac{m}{\Delta t} \frac{e^{2}}{n^{2} \hbar} \frac{e^{2}}{n \hbar}=\frac{m e^{4}}{\Delta t n^{3} \hbar^{2}}
$$

where

$$
\Delta v_{n}=\frac{e^{2}}{\hbar}\left(\frac{1}{n+1}-\frac{1}{n}\right) \approx-\frac{e^{2}}{\hbar n^{2}}
$$

is the velocity change along the track.

On the other hand the ratio $\frac{\Delta E}{\Delta t}$ can be approached on the basis of the formula [18]:

$$
\frac{\Delta E}{\Delta t}=e E_{e l} \frac{\Delta v_{n}}{2 \pi}=\frac{e^{6} m^{2}}{n^{4} \hbar^{4}} \frac{e^{2}}{\hbar n^{2}} \frac{1}{2 \pi}
$$

because for the quantum level $n$ we have the attractive force between the electron and nucleus equal to

$$
e E_{e l}=-\frac{e^{2}}{r_{n}^{2}}=-\frac{e^{2} m^{2} e^{4}}{n^{4} \hbar^{4}}=-\frac{e^{6} m^{2}}{n^{4} \hbar^{4}}
$$

and the change of velocity is reduced to direction normal to the electron orbit; see (43a). In effect of comparison done between (39) and (41) we obtain the following equation

$$
\frac{m e^{4}}{\Delta t n^{3} \hbar^{2}}=e E_{e l} \frac{\Delta v_{n}}{2 \pi} \cong \frac{e^{6} m^{2}}{n^{4} \hbar^{4}} \frac{e^{2}}{\hbar n^{2}} \frac{1}{2 \pi}=\frac{e^{8} m^{2}}{\hbar^{5} n^{6}} \frac{1}{2 \pi}
$$

because

$$
\frac{\Delta r_{n}}{\Delta t}=\frac{\Delta v_{n}}{2 \pi}
$$

This gives

$$
\Delta t=\frac{\hbar^{3} n^{3}}{m e^{4}} 2 \pi
$$

This last result can be compared with that obtained with the aid of the Joule-Lenz law. To this purpose we calculate the energy difference on the basis of the formula (39):

$$
\Delta E \cong \frac{m e^{4}}{n^{3} \hbar^{2}}
$$

The product of $\Delta E$ in (45) and $\Delta t$ in (44) gives:

$$
\Delta E \Delta t=\frac{m e^{4}}{n^{3} \hbar^{2}} 2 \pi \frac{\hbar^{3} n^{3}}{m e^{4}}=2 \pi \hbar=h .
$$

This result is in a perfect agreement with the product representing the Joule-Lenz law in (37).

An electrodynamical repercussion of the formulae (37) or (46) is very simple. Having $\Delta t$ we obtain the density of the electron charge current equal to

$$
i=\frac{e}{\Delta t} \text {. }
$$


On the other hand the potential $\Delta V$ connected with the energy change $\Delta E$ is equal to

$$
\Delta V=\frac{\Delta E}{e}
$$

In effect of the formulae (46), (46a) and (46b) the resistance of the current associated with $\Delta V$ and $i$ becomes:

$$
R=\frac{\Delta V}{i}=\frac{\Delta E}{e} \frac{\Delta t}{e}=\frac{h}{e^{2}}
$$

for any electron transition between two neighbouring quantum levels $n+1$ and $n$, on condition $n$ is a large number. The resistance $R$-equal to a well-known constant of the integer quantum Hall effect-is independent of $n$, so it can apply to any pair of large $n+1$ and $n$.

It seems of interest to perform a reversed procedure, i.e. to calculate $\Delta E$ on the basis of $R$ and $i$. This is obtained from the Joule formula

$$
\Delta E=R i^{2} \Delta t
$$

By substituting $R$ from (46c) and $i$ from (46a) we have

$$
\Delta E=\frac{h}{e^{2}} \frac{e^{2}}{\Delta t^{2}} \Delta t=\frac{h}{\Delta t}
$$

which is the same result as it was given in (37) and (46).

\section{Two Other Time Problems Entering the Quantum Physics}

Beyond of the time intervals characteristic for the electron transitions in the atom, two other special problems connected with time position in the quantum theory can be approached. The first one concerns the uncertainty problem of energy and time raised at the beginning of the modern quantum theory by Heisenberg [19]. This is represented by the relation

$$
\Delta E \Delta t>\hbar
$$

where $\Delta E$ and $\Delta t$ are respectively the energy and time intervals entering the quantum process. In this case the $\Delta E$ should not be confused with the interval specified in the formula (45).

The formula (47) - though formally similar to the uncertainty reference between the intervals of the particle momentum and position variables-was strongly criticized [20] [21] and omitted in numerous textbooks; see e.g. [22] [23]. In the author's opinion a more justified relation than (47) is [24] [25] [26]

$$
2 m c^{2} \Delta E(\Delta t)^{2}>\hbar^{2} \text {. }
$$

It can be noted that an application of (48) allowed us to obtain the relation between the fundamental constants of nature ( $e, h$ and $c)$; see [27].

Another special time problem which can be raised by the quantum theory is connected with the scale of time. A strictly progressive scale applied in general in physics is represented by Figure 1 .

This kind of scale means that any time $t_{B}$ later than time $t_{A}$ satisfies the 
relation

$$
t_{A}<t_{B} .
$$

However it can be shown that a different scale of time than given in Figure 1 is suitable when a perturbation of a stationary non-degenerate quantum state in some time moment, say $t_{A}$, is applied. Then the scale of time necessary to calculate the Schrödinger perturbation energy of order $N$-represented by a set of $N$ successive collisions of the original (unperturbed) state due to the perturbation potential $V^{\text {per }}$ - should be extended rather along a topological circle than a straight line.

In this case all energy terms belonging to the perturbation energy of order $N$, labelled in total by

$$
\Delta E_{N}
$$

can be readily obtained from the scale; see Figure 2 .

This is accomplished by taking into suitable contractions all $N-1$ time points

$$
t_{1}, t_{2}, \cdots, t_{N-2}, t_{N-1}
$$

present on the scale excepting for one point, say $t_{N}$. Then the set of the time loops created by contractions gives the corresponding set of the perturbed terms. The only rule which has to be applied to contractions is that the loops created by them should not cross [28] [29] [30].

\section{Summary and Conclusions}

In the paper, two problems of time entering respectively the special relativistic mechanics and quantum physics are outlined. In the first problem, an extremal calculation of the time difference between a moving and stationary frame of a one dimensional relativistic particle motion has been performed.

The extremal (minimal) time difference becomes equal to the ratio of the actual position variable $(z)$ and the velocity $(v)$ of the moving frame.

The time interval of the electron transition between the neighbouring quantum energy levels in the atom has been estimated in a semiclassical way. This

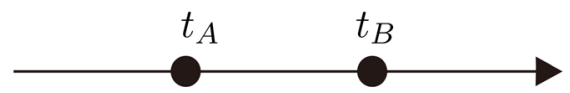

Figure 1. The time scale applied conventionally in physics.

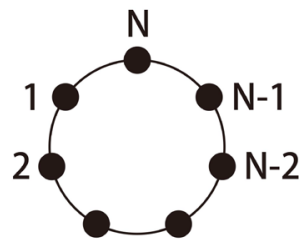

Figure 2. The time scale applied in course of examining the contributions to energy given by of the individual perturbation orders of the Schrodinger's theory. 
interval fits perfectly well the Joule-Lenz law coupling the energy difference between the levels to the value of the Planck constant.

As a second problem, a semiclassical calculation of the interval of time concerning the electron transition between the neighbouring quantum energy levels in the Bohr hydrogen atom has been performed.

In discussion, an extension of the original Heisenberg uncertainty principle for energy and time-criticized a time ago by several authors-has been presented. In a final step, we pointed out to the use of a circular scale of time-instead of a conventional straight-linear scale-in calculating the Schrödinger perturbation energy of a non-degenerate quantum state.

\section{Conflicts of Interest}

The author declares no conflicts of interest regarding the publication of this paper.

\section{References}

[1] Berliner, A. and Scheel, K. (1932) Physikalisches Handwörterbuch. 2nd Edition, Springer, Berlin. https://doi.org/10.1007/978-3-642-99643-6

[2] Landau, L.D. and Lifshits, E.M. (1948) Field Theory. OGIZ, Moscow. (In Russian)

[3] Sommerfeld, A. (1949) Mechanik. 4th Edition, Akademische Verlagsgesellschaft Geest \& Portig, Leipzig.

[4] Planck, M. (1932) Einführung in die Theorie der Wärme. S. Hirzel, Leipzig.

[5] Einstein, A. (1917) Zur Quantentheorie der Strahlung, Physikalische Zeitschrift, 18, 121-128.

[6] Slater, J.C. (1960) Quantum Theory of the Atomic Structure. Vol. 1, McGraw-Hill, New York

[7] Schiff, L.I. (1968) Quantum Mechanics. 3rd Edition, McGraw-Hill, New York.

[8] Bohr, N. (1924) Drei Aufsätze über Spektren und Atombau. 2nd Edition, Vieweg und Sohn, Braunschweig. https://doi.org/10.1007/978-3-642-64924-0_11

[9] Bohr, N. (1915) XXXVI. On the Series Spectrum of Hydrogen and the Structure of the Atom. The London, Edinburgh, and Dublin Philosophical Magazine and Journal of Science Series 6, 29, 332-335. https://doi.org/10.1080/14786440208635311

[10] Van der Waerden, B.L. (1967) Sources of Quantum Mechanics. Dover, New York.

[11] Sommerfeld, A. (1931) Atombau und Spektrallinien. Vol. 1, 5th Edition, Vieweg, Braunschweig.

[12] Lass, H. (1950) Vector and Tensor Analysis. McGraw-Hill, New York. https://doi.org/10.1119/1.1932684

[13] Olszewski, S. (2015) Non-Probabilistic Approach to the Time of Energy Emission in Small Quantum Systems. Journal of Modern Physics, 6, 1277-1288. https://doi.org/10.4236/jmp.2015.69133

[14] Olszewski, S. (2016) Quantum Aspects of the Joule-Lenz Law. Journal of Modern Physics, 7, 162-174. https://doi.org/10.4236/jmp.2016.71018

[15] Olszewski, S. (2016) Emission Intensity in the Hydrogen Atom Calculated from a Non-Probabilistic Approach to the Electron Transitions. Journal of Modern Physics, 7, 827-851. https://doi.org/10.4236/jmp.2016.78076 
[16] Olszewski, S. (2016) Semiclassical and Quantum Mechanical Formalism Applied in Calculating the Emission Intensity of the Atomic Hydrogen. Journal of Modern Physics, 1004-1020. https://doi.org/10.4236/jmp.2016.79091

[17] Olszewski, S. (2016) Erratum to "Semiclassical and Quantum-Mechanical Formalism Applied in Calculating the Emission Intensity of the Atomic Hydrogen" [Journal of Modern Physics 7 (2016) 1004-1020]. Journal of Modern Physics, 7, 2314-2315. https://doi.org/10.4236/jmp.2016.716199

[18] Landau, L.D. and Lifshits, E.M. (1969) Mechanics. Electrodynamics, Izd. Nauka, Moscow. (in Russian)

[19] Heisenberg, W. (1927) Über den anschaulichen Inhalt der quantentheoretischen Kinematik und Mechanik. Zeitschrift fuer Physik, 43, 172-198.

https://doi.org/10.1007/BF01397280

[20] Schommers, W. (1989) Space-Time and Quantum Phenomena. In: Schommers, W., Ed., Quantum Theory and Pictures of Reality, Springer, Berlin, 217-277. https://doi.org/10.1007/978-3-642-95570-9

[21] Bunge, M. (1970) The So-Called Fourth Indeterminacy Relation. Canadian Journal of Physics, 48, 1410-1411. https://doi.org/10.1139/p70-172

[22] Isaacs, A. (1990) Concise Dictionary of Physics. Oxford University Press, Oxford.

[23] Weinberg, S. (2013) Lectures on Quantum Mechanics. Cambridge University Press, Cambridge.

[24] Olszewski, S. (2011) Magnetic Field Induction and Time Intervals of the Electron Transitions Calculated on a Classical and Quantum-Mechanical Way. Journal of Modern Physics, 2, 1305-1309. https://doi.org/10.4236/jmp.2011.211161

[25] Olszewski , S. (2012) Relations between the Intervals Delta E and Delta t Obtained in the Deexcitaion Process of Electrons in Metlas. Journal of Modern Physics, 3, 217-220. https://doi.org/10.4236/jmp.2012.33030

[26] Olszewski, S. (2012) Intervals $\Delta \mathrm{E}$ and $\Delta \mathrm{t}$ Entering the Heisenberg Uncertainty Principle for Free Electrons and Their Limitations in the Magnetic Field. Quantum Matter, 1, 127-133. https://doi.org/10.1166/qm.2012.1010

[27] Olszewski, S. (2016) Relationship between the Fundamental Constants of Physics Obtained from the Uncertainty Principle for Energy and Time. Journal of Modern Physics, 6, 622-626. https://doi.org/10.4236/jmp.2015.65067

[28] Olszewski S., (2017) Circular Scale of Time and Construction of the Schrodinger Perturbation Series for Energy Made Simple. Journal of Modern Physics, 8, 1650-1684. https://doi.org/10.4236/jmp.2017.89098

[29] Olszewski, S. (2018) Circular Time Scale Yields a Recurrent Calculation of the Schrodinger Perturbation Energy. Journal of Modern Physics, 9, 1491-1521. https://doi.org/10.4236/jmp.2018.98093

[30] Olszewski, S. (2019) Circular Scale of Time as a Guide for the Schrödinger Perturbation Process of a Quantum-Mechanical System. World Journal of Mechanics, 9, 113-145. https://doi.org/10.4236/wjm.2019.95009 